www.jmscr.igmpublication.org

Impact Factor 5.244

Index Copernicus Value: 83.27

ISSN (e)-2347-176x ISSN (p) 2455-0450

crossref DOI:_http://dx.doi.org/10.18535/jmscr/v4i8.14

Journal Of Medical Science And Clinical Research

\title{
Predictors of Cognitive Impairment in Stroke Servivors
}

\author{
Authors \\ Gharib F. Mohamed ${ }^{1}$, HemedM. Baddary ${ }^{1}$, Mohamed N. Thabit ${ }^{1}$, A. Ammar Ghaibeh ${ }^{2}$, \\ Alaaeldin Sedky Bakhit Farrag ${ }^{1}$, Ahmad G. Arafa ${ }^{1}$ \\ ${ }^{1}$ Department of Neurolopsychiatry, Sohag University, Sohag, Egypt \\ ${ }^{2}$ Department of Medical Informatics, University of Tokushima, Tokushima, Japan \\ Corresponding Author \\ Mohamed Nasreldin Thabit Hamdoon \\ Neurology Department, Sohag University Hospital, Sohag University, Madinat Nasser, \\ Sohag, Egypt. Postal Code: 82524, Tel: +201004934646 \\ Email: Mohamed_hamdon@med.sohag.edu.eg
}

\begin{abstract}
The aim of this study is to determine the various risk factors and stroke characteristics that play a role in development of poststrokecongnitive impairment. A total of 119 chronic stroke patients were included in the study. Patients with history of psychiatric, medical or taking drugs that cause cognitive impairement were excluded from the study. The stroke patients were classified into normal or having mild, moderate and severe congnitiveimpairement according to the mini-mental state examination. Demographic variables and stroke risk factors including; age, sex, hypertension, diabetes mellitus, smoking, cardiac diseases, prior history of stroke or transient ischemic attacks were assessed. Various stroke symptomatology, history of delirium with the onset of stroke, and history of poststroke epilepsy were assessed.Barthel index and Beck depression scale were used to assess the global poststroke disability and poststroke depression. The lesion type, site, side, and size were assessed from the relevant brain imaging. The role of various previously mentioned factors in development of poststroke cognitive impairement were tested using the appropriate statistical test and C 4.5 decision tree for those factors were done.elderly stroke patients with multiple large sized strokes especially in the left hemisphere, those with cortical lesion, those associated with delirious onset, those who develop poststroke epilepsy and/or depression, and finally those patients with severe poststrokediseability are more likely to suffer psotstroke cognitive impairement.Early cognitive rehabilition methods and medical treatment with antidementia medications should be provided for patients with the associated previously mentioned factors.
\end{abstract}

Keywords: Stroke, Cognitive, Dementia, Predictors.

\section{INTRODUCTION}

Stroke is a major health problem that is associated with high mortality rates and morbidity in the survivors including motor, sensory, cognitive and psychiatric problems. Egypt is considered among the countries with high prevalence and incidence of stroke. The prevalence rate ranges from 5.6 to $9.6 / 1000$ and the annual incidence rate ranges from 1.8 to $2.5 / 1000$ population ${ }^{[1-3]}$.

Beside functional disabilities, chronic stroke patients and their caregivers frequently complain from various cognitive problems. In addition to 
the most common form "aphasia", these problems include memory impairment, mental slowness, defective executive functions, poor judgment, defective attention, and impaired visuos patial skills.

The frequency of post-stroke cognitive impairment ranges from $20 \%$ to $75 \%$ among stroke survivors ${ }^{[4,5]}$. These cognitive impairments may result in dramatic impact on the patient quality of life and substantially contributing to the overall morbidity. Most of the literature regarding this topic has originated from developed countries with little contribution from developing countries like Egypt .

As the cost of post-stroke care is directly related to post-stroke cognitive decline, clear understanding of the factors associated with poststroke cognitive impairment is of great importance. Many sociodemographic, clinical, and imaging variables has been treated as determinants of post-stroke cognitive decline including lesion location ${ }^{[6]}$, lesion size, diabetes mellitus, time elapsed from the onset of stroke, age and gender and many others ${ }^{[7]}$. Despite the amble amount of literature, there is still unclear conclusion about the factors associated with the post-stroke cognitive decline ${ }^{[6]}$. Post-stroke depression was found to be positively correlated with cognitive impairment in many studies ${ }^{[8]}$, however few studies did not confirm this relation.

The method of assessment of post-stroke cognitive decline seems to be an extremely important issue. Many studies used the Mini Mental State Examination (MMSE) as a method for global assessment of cognitive functions which is a more general estimate of various domains of cognitive function. However, other studies used more sophisticated neuropsychological batteries which are better than the MMSE in the diagnosis of the type of cognitive impairment. The disadvantages of those sophisticated tests include the unavailability in most health care centers, difficulty for assessment of all the neuropsychological functions in more severely affected individuals, and finally those tests are time consuming and not easy to do if compared to the MMSE ${ }^{[9]}$.

Decision trees are powerful and accurate prediction methods that have been successfully used in various medical aspects including stroke and head ${ }^{[10]}$. Decision trees may be more accurate that the classical logistic regression model if compared together .Different from classical statistical methods that assume that factors exert the same effect over the whole data set, decision trees recursively divide data into subgroups and investigate the effects of different factors based on their statistical importance for different subgroups.

According to the previous description, what are the various risk factors that may play a role in the development of post-stroke global cognitive decline?, and how can we early predict the possibility for development of post-stroke global cognitive decline?. We need answers to these questions. The answer of these questions is very important to be able for setting a more comprehensive management plan for these patients. In this study, we measured the global post-stroke cognitive impairment and its relation to various socioecodemographic, stroke risk factors, clinical stroke symptomatology, and radiological factors. Moreover, we tried to set a prediction model through data mining procedure as a step forward for a more comprehensive approach for early prediction of post-stroke global cognitive impairment.

\section{MATERIALS AND METHODS}

We retrospectively reviewed a total of 119 chronic stroke patients who were examined in Sohag University Hospital, Department of Neurology between May 2012 and April 2013. Written informed consent was signed by the patient or one of his relatives. The study protocol was approved by local ethics committee of Sohag University Hospital

\section{Patient selection criteria}

Patients with chronic stroke defined as stroke 
duration > 1 year were screened for inclusion in the study. Stroke was defined according to the WHO definition as "rapidly developing clinical signs of focal (or global) disturbance of cerebral function, with symptoms lasting for 24 hours or longer, with no apparent cause other than vascular origin". The diagnosis was confirmed by CT/MRI brain. Both ischemic and hemorrhagic stroke were included in the study. We excluded patients in acute and subacute phase of stroke (patients with stroke duration $<1$ year), patients with subarachnoid hemorrhage, patients with prior history of psychiatric disorders as major depression or schizophrenia patients with history of degenerative neurological or medical disorders that affect cognitive functions, patients with severe neurological disabilities that impairs communication such as aphasia, and patients in whom the brain imaging is not clear enough to assess the diagnosis as early imaging without apparent lesions and those with CT/MRI not clear enough to assess the size of stroke lesion.

\section{Patient evaluation}

All patients included in the study were subjected to detailed medical history and neurological examination. Demographic variables as age and sex were noted. The various stroke main risk factors were assessed including hypertension, diabetes mellitus, smoking, cardiac diseases, and prior history of transient ischemic attacks or previous stroke. Stroke symptomatology was noted including right and left hemiparesis/ hemiplegia, dysarthria, hemi-sensory loss/heminumbness, vertigo/hemiataxia, delirium with the onset of stroke, and post-stroke epilepsy were noted. The stroke duration in months was also taken.

\section{Brain Imaging}

We assessed the available brain imaging either CT brain and/or MRI and gathered the available information about the nature of stroke lesion including the lesions type (infarction/ hemorrhage), lesion side (left hemispheric/right hemispheric or cerebellar or brainstem), and lesion number. The location of the lesion was categorized into pure subcortical stroke which includes lesion in the following site: basal ganglia/internal capsule, thalamic, brainstem, and cerebellar, and finally strokes that include cortical distribution up to hemispheric surface. The size of the lesion was detected by measuring its largest anteroposterior diameter (A) and its largest horizontal diameter (B) with a caliper in millimeter. The third, vertical diameter (C) was determined by summing the thicknesses of the slices in which the lesion was visible. The lesion volume was calculated according to the formula $0.5 \times \mathrm{AxB} \times \mathrm{C}^{[11]}$.

\section{Global post-stroke disability and global cognitive abilities}

Different standard neurological scales were measured to assess various post-stroke disabilities in this whole project including scales to assess cognitive, motor, and balance functions in addition to psychiatric state, namely depression. As a measure for global cognitive abilities in poststroke patients, MMSE was used. The MMSE score was graded as normal (score $\geq 27$ ), Mild cognitive impairment (score 21-26), moderate cognitive impairment (score 11-20), and severe cognitive impairment (score $\leq 10$ ). For statistical analysis we classified the patients into 3 groups: normal (score $\geq 27$ ), mild cognitive impairment (score 21-26), moderate to severe cognitive impairment (score $\leq 20$ ). Arabic validated version of beck depression score was used for assessment of post-stroke depression with a total score of 63 points[12]. The Barthel Index (BI) was used as a scoring technique that measures the overall stroke disability by assessing the patient's performance in 10 activities of daily life. The items can be divided into a group that is related to self-care (feeding, grooming, bathing, dressing, bowel and bladder care, and toilet use) and a group related to mobility (ambulation, transfers, and stair climbing). The maximal score is 100 if 5-point increments are used, indicating that the patient is 
fully independent in physical functioning. The lowest score is 0 , representing a totally dependent bedridden state.

\section{Decision tree}

Decision trees are classification algorithms widely used in data mining because there results are easily understandable. A decision tree is generated by recursively splitting the patients according to the values of a statistically important factors. The C4.5 Decision Tree used in this work uses information gain ratio to determine the statistically important factors. The tree was implemented using RapidMiner 5.3 software in combination with feature selection. The overall accuracy, sensitivity, and specificity was calculated using 10-folds cross validation method.

\section{Statistical Analysis}

Categorical data were presented as number (n) and percentage $(\%)$, while continuous variables were presented as mean \pm standard error of mean (SEM) if normally distributed and median (range) if abnormally distributed as tested by Kolmogorov-Smirnov Z for normality. Categorical data were analyzed using Chi-square test, while student t-test or one way ANOVA was used to compare means for normally distributed two or more groups of continuous variables if normally distributed. Mann-Whitney $U$ and Kruskal-Wallis tests were used if data are not normally distributed. P-value $\leq 0.05$ was considered statistically significant. Multinomial logistic regression was used as multivariate analysis for independent assessment of the factors associated with the global cognitive decline in chronic stroke patients. The Statistical Package for Social Sciences (SPSS) version 16 was used for statistical analyses.

\section{RESULTS}

Table (1) presents the demographic, stroke risk factors, stroke symptomatology, and imaging data of the study participants. Taking the all factors in consideration, we found significant difference in MMSE groups in relation to the number of strokes (either single or recurrent), location of stroke (either subcortical or cortical), number of CT/MRI lesions (one lesion, two lesions or > two lesions), dysarthria, delirium with stroke onset, and poststroke epilepsy (table 2). For patients with dysarthria, it seems that those patients have less tendency to develop cognitive impairementas all cases with dysarthria were either normal or suffer mild global cognitive impairment. Both Beck depression scale was significantly different between normal, mild and moderate to severe global cognitive impairment $(\mathrm{F}=5.174, \mathrm{P}=$ 0.007). Post-hoc t-test revealed statistically significant difference between normal and mild cognitive impairment, and normal and moderate to severe cognitive impairment $(\mathrm{P}=0.038$; and $\mathrm{P}=$ 0.004) Fig.(1). For BI, KruskalWillas test revealed a significant difference between normal, mild and moderate to severe global cognitive impairment $\left(\alpha^{2}=10.4, \mathrm{P}=0.006\right)$. Post-hoc testing revealed significant difference between normal and mild cognitive impairment, and normal and moderate to severe cognitive impairment $(\mathrm{P}=0.01$; and $\mathrm{P}=$ 0.008) Fig. (2).

To determine the factors that are independently associated with development of global cognitive impairment in our study, multivariate statistics, namely multinomial logistic regression taking normal MMSE as reference category and mild and moderate to severe MMSE impairment as test categories for the factors which were significant in univariate statistics was done. For stroke times, both single stroke and recurrent stroke have more probability to develop mild and moderate to severe global cognitive impairment after stroke compared to normal MMSE group $(\mathrm{p}=0.001$; OR $=0.128 ; 95 \% \mathrm{CI}=0.039-0.421$, and $\mathrm{p}=0.002$; $\mathrm{OR}=0.152 ; 95 \% \quad \mathrm{CI}=0.047-0.492$ ) respectively. The same applies for lesion number as both single lesion and multiple lesions have more probability to develop mild and moderate to severe global cognitive impairment after stroke compared to normal MMSE group $(p=0.001$; OR 


\section{JMSCR Vol||04||Issue||08||Page 11771-11781||August}

$=0.179 ; 95 \% \mathrm{CI}=0.065-0.492$, and $\mathrm{p}=0.003$; $\mathrm{OR}=0.22 ; 95 \% \mathrm{CI}=0.082-0.589$ ) respectively. However delerium with onset, post-stroke epilepsy, and lesion location increase the probability of developing moderate to severe global cognitive impairment rather than mild global cognitive impairment if compared to normal MMSE group $(\mathrm{p}=0.024 ; \mathrm{OR}=0.348$; $95 \% \mathrm{CI}=0.139-0.868$, and $\mathrm{p}=0.002 ; \mathrm{OR}=$ $0.191 ; 95 \% \mathrm{CI}=0.065-0.558$, and $\mathrm{p}=0.029$; $\mathrm{OR}=4.286 ; 95 \% \mathrm{CI}=1.161-15.824)$ respectively. For Beck depression and BI, multinomial logistic regression revealed both of them increased the probability to induce mild and moderate to severe global cognitive impairment after stroke compared to normal MMSE group (p $=0.038 ; \mathrm{OR}=0.986 ; 95 \% \mathrm{CI}=0.973-0.999$, and $\mathrm{p}=0.004 ; \mathrm{OR}=0.981 ; 95 \% \mathrm{CI}=0.968-$ 0.994 for Beck depression scale and $\mathrm{p}=0.009$; $\mathrm{OR}=1.053 ; 95 \% \mathrm{CI}=1.013-1.095$, and $\mathrm{p}=$ $0.012 ; \mathrm{OR}=1.049 ; 95 \% \mathrm{CI}=1.011-1.089$ for $\mathrm{BI})$ respectively.

The results of the decision trees was classified according to the lesion number. Fig.(3) and Fig. (4)showed the decision trees for both multiple brain lesions and single brain lesion in our stroke patients.

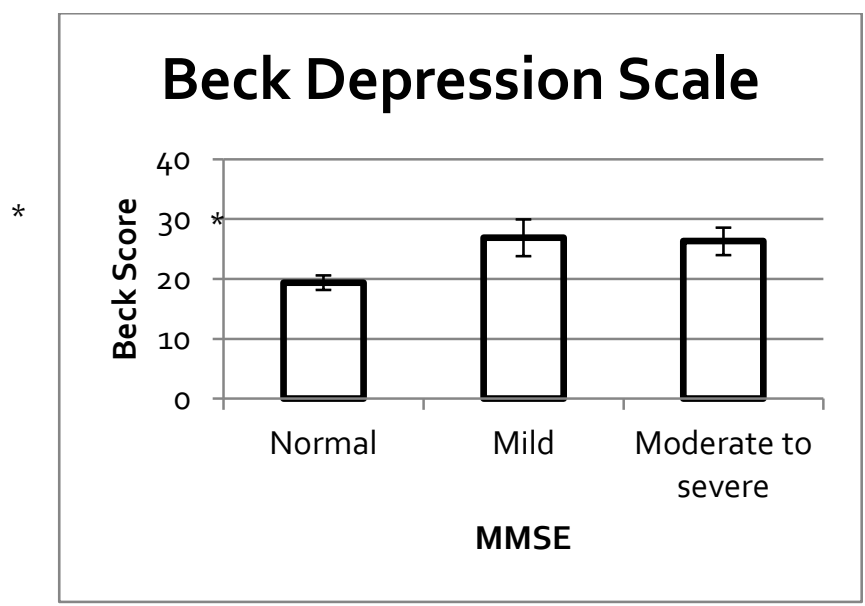

Figure 1: Effect of poststroke depression on poststroke cognitive impairement. There were significant differences between normal and mild cognitive impairment, and normal and moderate to severe cognitive impairment as regard Beck depression score

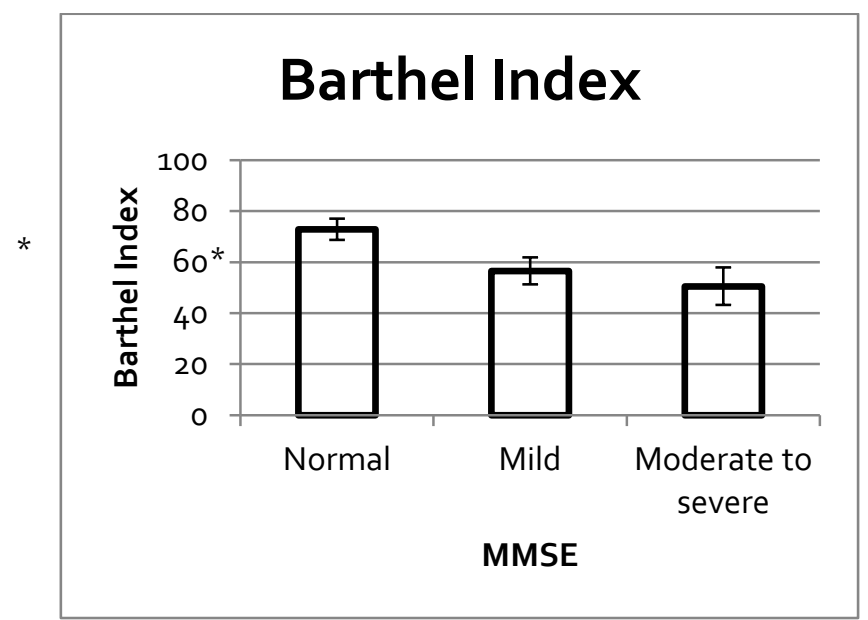

Figure 2: Effect of poststroke overall disability on poststroke cognitive impairement. There were significant differences between normal and mild cognitive impairment, and normal and moderate to severe cognitive impairment as regard Barthel index score 


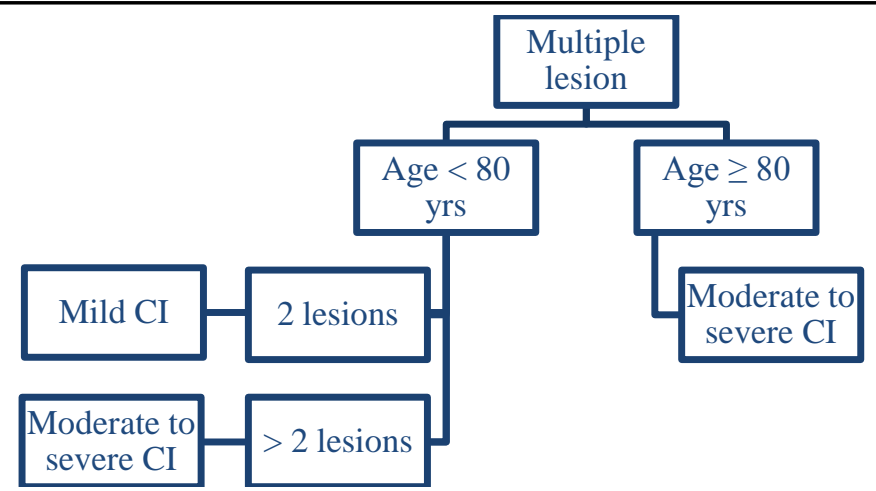

Figure 3: Decision tree for patients with multiple brain lesions.Yrs $=$ Years, and $\mathrm{CI}=$ Cognitive Impairement

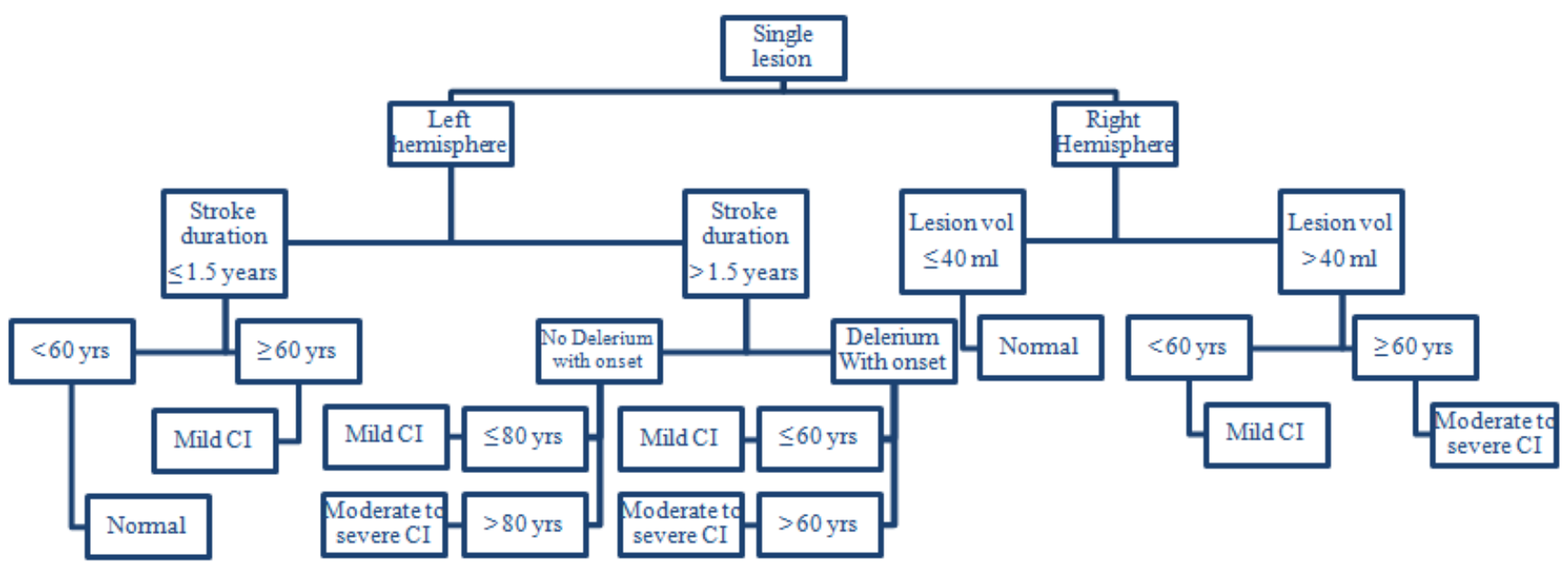

Figure 4: Decision tree for single brain lesion. Yrs = Years, and CI = Cognitive Impairement

Table (1): demographic, stroke risk factors, stroke symptomatology, and imaging data of the study participants

\begin{tabular}{lll}
\hline Variant & & Frequency \\
\hline Demographic variables & & \\
\hline Age & (Mean \pm SEM) & $58.97 \pm 0.92$ \\
\hline Sex & Male & $68(57.1 \%)$ \\
\cline { 2 - 3 } & Female & $51(42.9 \%)$ \\
\hline Stroke variables including & imaging data & \\
\hline Stroke type & Ischemic stroke & $97(81.5 \%)$ \\
\cline { 2 - 3 } & Hemorrhagic stroke & $22(18.5 \%)$ \\
\hline Stroke times & Single & $92(77.3 \%)$ \\
\cline { 2 - 3 } & Recurrent & $27(22.7 \%)$ \\
\hline Stroke duration & Median (Range) & $30(163)$ \\
\hline Brain lesion Side & Rt hemisphere/cerebellum & $56(47.1 \%)$ \\
\cline { 2 - 3 } & Lt hemisphere/cerebellum & $62(52.1 \%)$ \\
\cline { 2 - 3 } & Brainstem (pontine) & $1(0.8 \%)$ \\
\hline Brain lesion Side & Rt hemisphere/cerebellum & $56(47.1 \%)$ \\
\cline { 2 - 3 } & Lt hemisphere/cerebellum & $62(52.1 \%)$ \\
\cline { 2 - 3 } & Brainstem (pontine) & $1(0.8 \%)$ \\
\hline Lesion number & 1 & $81(68.1 \%)$ \\
\cline { 2 - 3 } & 2 & $28(23.5 \%)$ \\
\cline { 2 - 3 } & $>2$ & $24(20.4 \%)$ \\
\hline Lesion location & Basal ganglia/internal capsule/thalamus & $27(73.1 \%)$ \\
\cline { 2 - 3 } & Cortical & $7(5.9 \%)$ \\
\cline { 2 - 3 } & Cerebellar & \\
\hline
\end{tabular}




\begin{tabular}{|c|c|c|}
\hline & Brainstem & $1(0.8 \%)$ \\
\hline \multirow{2}{*}{$\begin{array}{l}\text { Compliance to } \\
\text { antiplatelet therapy }\end{array}$} & Regular & $44(44.1 \%)$ \\
\hline & Irregular & $54(55.1 \%)$ \\
\hline \multicolumn{3}{|l|}{ Stroke risk factors } \\
\hline \multirow[t]{2}{*}{ Smoking } & Non smoker & $58(48.7 \%)$ \\
\hline & Smoker & $61(51.3 \%)$ \\
\hline \multirow[t]{2}{*}{ Hypertension } & Normotensive & $20(16.8 \%)$ \\
\hline & Hypertensive & $99(83.2 \%)$ \\
\hline \multirow[t]{2}{*}{ Diabetes } & Non diabetic & $71(59.7 \%)$ \\
\hline & Diabetic & $48(40.3 \%)$ \\
\hline \multirow[t]{3}{*}{ Heart disease } & Non cardiac & $86(72.3 \%)$ \\
\hline & IHD & $19(16 \%)$ \\
\hline & RHD & $14(11.8 \%)$ \\
\hline \multirow[t]{2}{*}{ Previous TIA } & No TIAs & $114(95.8 \%)$ \\
\hline & TIAs & $5(4.2 \%)$ \\
\hline \multicolumn{3}{|l|}{ Stroke symptomatology } \\
\hline \multirow[t]{8}{*}{ Symptom/sign } & Lt Hemiplegia/Hemiparesis & $64(53.8 \%)$ \\
\hline & Rt Hemiplegia/Hemiparesis & $56(47.1 \%)$ \\
\hline & Dysarthria & $10(8.4 \%)$ \\
\hline & Hemi-sensory loss or numbness & $3(2.5 \%)$ \\
\hline & Delirium with onset & $36(30.3 \%)$ \\
\hline & Vertigo/ataxia & $13(10.9 \%)$ \\
\hline & Behavioral changes & $3(2.5 \%)$ \\
\hline & Post-stroke epilepsy & $22(18.5 \%)$ \\
\hline
\end{tabular}

Table (2): Statsitical analysis of the various factors related to poststroke cognitive impairment

\begin{tabular}{|c|c|c|c|c|c|}
\hline \multirow[b]{2}{*}{ Variant } & & \multirow{2}{*}{$\begin{array}{l}\text { Normal } \\
\text { MMSE } \\
\mathbf{N}=\mathbf{6 2}\end{array}$} & \multicolumn{2}{|c|}{ Impaired MMSE } & \multirow[b]{2}{*}{ P-value } \\
\hline & & & $\begin{array}{l}\text { Mild } \\
\mathrm{N}=27\end{array}$ & $\begin{array}{l}\text { Moderate to } \\
\text { severe } \mathrm{N}=30\end{array}$ & \\
\hline \multicolumn{6}{|c|}{ Demographic variables } \\
\hline \multicolumn{2}{|l|}{ Age $($ Mean \pm SEM $)$} & $58.63(1.3)$ & $56.81(1.9)$ & $61.60(1.6)$ & 0.185 \\
\hline \multirow{2}{*}{ Sex } & Male & $38(61.3 \%)$ & $14(51.9 \%)$ & $16(53.3 \%)$ & \multirow{2}{*}{0.387} \\
\hline & Female & $24(38.7 \%)$ & $13(48.1 \%)$ & $14(46.7 \%)$ & \\
\hline \multicolumn{6}{|c|}{ Stroke variables including imaging data } \\
\hline \multirow{2}{*}{ Stroke type } & Ischemic stroke & $48(77.4 \%)$ & $25(92.6 \%)$ & $24(80 \%)$ & \multirow{2}{*}{0.453} \\
\hline & Hemorrhagic stroke & $14(22.6 \%)$ & $2(7.4 \%)$ & $6(20 \%)$ & \\
\hline \multirow{2}{*}{ Stroke times } & Single & $57(91.9 \%)$ & $16(59.3 \%)$ & $19(63.3 \%)$ & \multirow{2}{*}{$\begin{array}{l}< \\
0.0001\end{array}$} \\
\hline & Recurrent & $5(8.1 \%)$ & $11(40.7 \%)$ & $11(36.7 \%)$ & \\
\hline \multicolumn{2}{|c|}{ Stroke duration(Median (Range) } & $30(126)$ & $24(79)$ & $31(162)$ & 0.337 \\
\hline \multirow{3}{*}{ Brain lesion Side } & Rt hemisphere/cerebellum & $31(50 \%)$ & $9(33.3 \%)$ & $16(53.3 \%)$ & \multirow{2}{*}{0.774} \\
\hline & Lt hemisphere/cerebellum & $30(48.4 \%)$ & $18(66.7 \%)$ & $14(46.7 \%)$ & \\
\hline & Brainstem (pontine) & $1(1.6 \%)$ & $0(0 \%)$ & $0(0 \%)$ & \\
\hline \multirow{3}{*}{ Lesion number } & 1 & $52(83.9 \%)$ & $13(48.1 \%)$ & $16(53.3 \%)$ & \multirow{3}{*}{0.0001} \\
\hline & 2 & $5(8.1 \%)$ & $9(33.3 \%)$ & $14(46.7 \%)$ & \\
\hline & $>2$ & $5(8.1 \%)$ & $5(18.5 \%)$ & $0(0 \%)$ & \\
\hline Lesion location & Pure Subcortical & $21(33.9 \%)$ & $9(33.3 \%)$ & $3(10 \%)$ & 0.043 \\
\hline
\end{tabular}




\begin{tabular}{|c|c|c|c|c|c|}
\hline & Cortical & $41(66.1 \%)$ & $18(66.3 \%)$ & $27(90 \%)$ & \\
\hline \multicolumn{2}{|c|}{ Lesion Volume in ML (Median (range)) } & $8.1(285.7)$ & $5.4(105.2)$ & $16.4(556.5)$ & 0.086 \\
\hline \multirow{2}{*}{$\begin{array}{l}\text { Compliance to } \\
\text { antiplatelet therapy in ischemic } \\
\text { stroke cases }\end{array}$} & Regular & $21(43.8 \%)$ & $9(36 \%)$ & $14(58.3 \%)$ & \multirow{2}{*}{0.278} \\
\hline & Irregular & $27(56.2 \%)$ & $16(64 \%)$ & $10(41.7 \%)$ & \\
\hline \multicolumn{6}{|l|}{ Stroke risk factors } \\
\hline Smoking & Smoker & $61(51.3 \%)$ & $12(44.4 \%)$ & $18(60 \%)$ & 0.523 \\
\hline Hypertension & Hypertensive & $52(83.9 \%)$ & $22(81.5 \%)$ & $25(83.3 \%)$ & 0.891 \\
\hline Diabetes Mellitus & Diabetic & $24(38.7 \%)$ & $9(33.3 \%)$ & $15(50 \%)$ & 0.449 \\
\hline Previous TIAs & Previous TIAs & $5(8.1 \%)$ & $0(0 \%)$ & $0(0 \%)$ & 0.091 \\
\hline \multirow{3}{*}{ Heart disease } & Normal & $40(64.5 \%)$ & $23(85.2 \%)$ & $23(76.7 \%)$ & \multirow{3}{*}{0.234} \\
\hline & IHD & $14(22.6 \%)$ & $0(0 \%)$ & $5(16.7 \%)$ & \\
\hline & RHD & $8(12.9 \%)$ & $4(14.8 \%)$ & $2(6.7 \%)$ & \\
\hline
\end{tabular}

Stroke symptomatology

\begin{tabular}{l|l|l|l|l|l}
\hline \multirow{4}{*}{ Symptom/sign } & Lt Hemiplegia/Hemiparesis & $35(56.5 \%)$ & $12(44.4 \%)$ & $17(56.7 \%)$ & 0.542 \\
\cline { 2 - 6 } & Rt Hemiplegia/Hemiparesis & $26(41.9 \%)$ & $17(63 \%)$ & $13(43.3 \%)$ & 0.169 \\
\cline { 2 - 6 } & Dysarthria & $4(6.5 \%)$ & $5(18.5 \%)$ & $0(0 \%)$ & $\mathbf{0 . 0 2 8}$ \\
\cline { 2 - 6 } & Hemi-sensory loss or numbness & $3(4.8 \%)$ & $0(0 \%)$ & $0(0 \%)$ & 0.243 \\
\hline \multirow{3}{*}{} & Delirium with onset & $16(25.8 \%)$ & $5(18.5 \%)$ & $15(50 \%)$ & $\mathbf{0 . 0 1 9}$ \\
\cline { 2 - 6 } & Vertigo/ataxia & $4(6.5 \%)$ & $4(14.8 \%)$ & $1(3.3 \%)$ & 0.234 \\
\hline & Behavioral changes & $1(1.6 \%)$ & $1(3.7 \%)$ & $1(3.3 \%)$ & 0.802 \\
\cline { 2 - 6 } & Post-stroke epilepsy & $7(11.3 \%)$ & $3(11.1 \%)$ & $12(40 \%)$ & $\mathbf{0 . 0 0 2}$ \\
\hline
\end{tabular}

\section{Other Factors}

\begin{tabular}{l|l|l|l|l|l}
\hline Post-stroke Depression & $\begin{array}{l}\text { Beck Depression Scale (mean } \pm \\
\text { SEM) }\end{array}$ & $19.4 \pm 1.2$ & $26.9 \pm 3.1$ & $26.3 \pm 2.3$ & $\mathbf{0 . 0 0 6}$ \\
\hline Overall Stroke Disability & Barthel index (Median (Range)) & $90(95)$ & $55(85)$ & $52.5(100)$ & $\mathbf{0 . 0 0 7}$ \\
\hline
\end{tabular}

\section{DISCUSSION}

We found that patients who have single stroke with single brain lesion have more probability to develop mild cognitive impairment compared to those with recurrent strokes and multiple lesions. However, elderly patients with recurrent strokes with multiple large brain lesions especially of the left hemisphere compared to single stroke with single small brain lesion, those who suffers delirium with the onset of stroke compared to those without delirious onset, those who develop epileptic seizures after stroke compared to those without epileptic seizures andthose with cortical strokes compared with subcortical strokes are significantly more likely to develop moderate to severe cognitive impairment. The development of post stroke depression and the high level of overall post stroke disability are significantly more likely to develop both mild and moderate to severe cognitive impairment in the chronic stroke phase. The sex of the patients, stroke type (ischemic or hemorrhagic), compliance to antiplatelet therapy, smoking, hypertension, diabetes mellitus, history of previous TIAs, and heart diseases did not significantly influenced the development of post stroke cognitive impairment weather mild or moderate to severe in our study. The main objective of our cross-sectional study was to determine the risk factors of post-stroke cognitive impairement in anrandom sample of registered stroke patients. Age and duration may have an impact on the development of post stroke 
cognitive impairment, however we didn't find statistically significant difference as regard the age in patients with and without post-stroke cognitive impairement in our study. This was in agreement with and the Framingham study ${ }^{[4]}$. Although the standard statistical methods revealed no significant effect of age in developing psotstroke cognitive impairment, handling our data again using more accurate predictive methods of analysis as decision trees revealed the extreme importance of age as a strong factor for development of poststroke cognitive impairement as in agreement with other studies ${ }^{[13,14]}$ which agree thatthe risk of post-stroke cognitive impairement increases with advanced age. Recently,decreased total cerebral blood flow and the reduced proportional aqueductal and cervical cerebrospinal fluid pulsations are the result of arterial loss of pulsatility may be causes of cognitive decline with age ${ }^{[15]}$.

Also, no sex differences in patients with and without post-stroke cognitive impairement in our study and this was similar to the results of other studies $^{[4,5]}$. The increased risk of dementia in older females is consistent with some studies ${ }^{[16]}$.This variation may be due to racial differences and estrogen depletion with age which increases the incidence of cognitive decline in some women.

In our study,post-stroke cognitive impairement was unrelated to type of stroke (ischemic or hemorrhagic), sideof brain lesion, and the volume of brain lesion. This was in agreement with other studies $^{[5]}$. While in the study of Khedr et al.poststroke cognitive impairement was more associated with large infarcts, lacunar infarcts and dominant hemispheric lesions ${ }^{[13]}$. The same situation happens again, as the standard statistical methods used in handling our date failed to show the effect of lesion size and hemispheric location on development of psotstroke cognitive imparement, decision trees proved the effect of those important factors for development of poststroke cognitive impairement. One more thing to explain why the usual statistical methods in our study did not show same results as Khedr etal. is the use of more sophsticaed test batteries and event related potentials in the mentioned study, however the aim of our study is to assess the global cognitive impairement rather thant the detailed analysis of each cognitive domain.

In our study, poststroke cognitive impairement was significantly associated with increased lesion number and stroke times. This was in agreement with many studies.Vinters and colleagues described three pathogenic concepts of vascular cognitive impairement based on pathological and functional imaging data including the accumulated cortical infarcts, the strategic subcortical infarcts and the functional cortical disconnection ${ }^{[17]}$.

In the present study, high blood pressure, diabetes mellitus, transient ischemic attacks, current smoking, and heart diseases are not associated with poststroke cognitive impairement. This was similar to the results of other studies ${ }^{[5]}$. Hypertension ${ }^{[13]}$, diabetes,smoking ${ }^{[13]}$ andmyocardi al infarction were found to predict poststroke cognitive impairement in somestudies but not in others. The relation between the previously mentioned factors and poststroke cognitive impairement is controversial among various literature, according further studies are needed to further explain the role of those various stroke risk factors in the development of poststroke cognitive impairement.

In our study, poststroke epilepsy was significantly associated with poststroke cognitive impairement and this was similar to the results found by other studies ${ }^{[18]}$. It seems that seizure occurrence in poststroke patients is not related to the severity of the stroke but rather to the degree of cognitive impairment indicating that seizures occurrence is more probably due to underlying neurodegenerative process responsible for the mental deterioration.

In our study, patients with high level of overall post stroke disability are significantly more likely to develop both mild and moderate to severe cognitive impairment in the chronic stroke phase. This was consistent with several studies ${ }^{[5]}$. Also in our study, delirium in acute stroke phase was 
significantly associated with poststroke cognitive impairment and this was similar to some studies [19]. We supposed that delirium and dementia have a close, bidirectional relationship. Dementia is a risk factor for delirium, whereas delirium may be an early sign of emerging dementia.

Post-stroke depression was found to be positively correlated with cognitive impairment in our study and this was in agreement with several studies[8], however few studies did not confirm this relation.Some studies supposed that lesions in the deep brain structures may contribute to depression among older persons "vascular depression hypothesis". Also, Minett et al.found thatthe presence of a history of late-onset depression was a strong correlate of white matter lesion severity, even after adjusting for age, gender, and education $^{[20]}$.

\section{CONCLUSION}

In conclusion, taking the results of our study and the other various literatures that addressed the factors related to the poststroke cognitive impairement in consideration we can say that elderly rather than young stroke patients, those with multiple strokes rather than single stroke, those with multiple brain lesiosns, those with large sized brain lesions especially in the left hemisphere, those with cortical rather than subcortical brain lesions, those associated with delirious onset, those who develop poststroke epilepsy and/or depression, and finally those patients with severe poststrokediseability are more likely to suffer psotstroke cognitive impairement. Early cognitive rehabilition methods and medical treatment with antidementia medications should be provided for those patients. Larger multicenter studies are need with the used of more sophisticated methods of assessment of cognitive functions including neuropsychological computerized test batteries, cognitive neurophysiological methods, and functional neuroimaging methods are needed to futher explain the important factors that might play a role in development of poststroke cognitive impairment.

\section{REFRENCES}

1. El-Tallawy, H.N., W.M. Farghaly, G.A. Shehata, N.M. Abdel-Hakeem, T.A. Rageh, R. Badry, and M.R. Kandil, Epidemiology of non-fatal cerebrovascular stroke and transient ischemic attacks in Al Quseir, Egypt. Clin Interv Aging, 2013. 8: p. $1547-51$.

2. Farghaly, W.M., H.N. El-Tallawy, G.A. Shehata, T.A. Rageh, N.M. AbdelHakeem, M.A. Elhamed, B.M. Al-Fawal, and R. Badry, Epidemiology of nonfatal stroke and transient ischemic attack in AlKharga District, New Valley, Egypt. Neuropsychiatr Dis Treat, 2013. 9: p. 1785-90.

3. Khedr, E.M., N.A. Elfetoh, G. Al Attar, M.A. Ahmed, A.M. Ali, A. Hamdy, M.R. Kandil, and H. Farweez, Epidemiological study and risk factors of stroke in Assiut Governorate, Egypt: community-based study. Neuroepidemiology, 2013. 40(4): p. 288-94.

4. Ivan, C.S., S. Seshadri, A. Beiser, R. Au, C.S. Kase, M. Kelly-Hayes, and P.A. Wolf, Dementia after stroke: the Framingham Study. Stroke, 2004. 35(6): p. 1264-8.

5. Barba, R., S. Martinez-Espinosa, E. Rodriguez-Garcia, M. Pondal, J. Vivancos, and T. Del Ser, Poststroke dementia : clinical features and risk factors. Stroke, 2000. 31(7): p. 1494-501.

6. Spalletta, G., D. De Angelis, and C. Caltagirone, Poststroke depression and emotional incontinence. Neurology, 2000. 55(11): p. 1760-1.

7. Kim, J.S. and S. Choi-Kwon, Poststroke depression and emotional incontinence: correlation with lesion location. Neurology, 2000. 54(9): p. 1805-10.

8. Lamb, F., J. Anderson, M. Saling, and H. Dewey, Predictors of subjective cognitive complaint in postacute older adult stroke patients. Arch Phys Med Rehabil, 2013. 94(9): p. 1747-52. 
9. Spalletta, G., G. Guida, D. De Angelis, and C. Caltagirone, Predictors of cognitive level and depression severity are different in patients with left and right hemispheric stroke within the first year of illness. $\mathrm{J}$ Neurol, 2002. 249(11): p. 1541-51.

10. Marrega, L.H., S.M. Silva, E.F. Manffra, and J.C. Nievola, Comparison between Decision Tree and Genetic Programming to distinguish healthy from stroke postural sway patterns. Conf Proc IEEE Eng Med Biol Soc, 2015. 2015: p. 6820-3.

11. Pantano, P., F. Caramia, L. Bozzao, C. Dieler, and R. von Kummer, Delayed increase in infarct volume after cerebral ischemia: correlations with thrombolytic treatment and clinical outcome. Stroke, 1999. 30(3): p. 502-7.

12. Abdel-Khalek, A.M., Internal consistency of an Arabic Adaptation of the Beck Depression Inventory in four Arab countries. Psychol Rep, 1998. 82(1): p. 264-6.

13. Khedr, E.M., S.A. Hamed, H.K. ElShereef, O.A. Shawky, K.A. Mohamed, E.M. Awad, M.A. Ahmed, G.A. Shehata, and M.A. Eltahtawy, Cognitive impairment after cerebrovascular stroke: Relationship to vascular risk factors. Neuropsychiatr Dis Treat, 2009. 5: p. 103-16.

14. Douiri, A., A.G. Rudd, and C.D. Wolfe, Prevalence of poststroke cognitive impairment: South London Stroke Register 1995-2010. Stroke, 2013. 44(1): p. 138-45.

15. Stoquart-ElSankari, S., O. Baledent, C. Gondry-Jouet, M. Makki, O. Godefroy, and M.E. Meyer, Aging effects on cerebral blood and cerebrospinal fluid flows. J Cereb Blood Flow Metab, 2007. 27(9): p. 1563-72.

16. Farrag, A.K., E.M. Khedr, H. AbdelAleem, and T.A. Rageh, Effect of surgical menopause on cognitive functions. Dement Geriatr Cogn Disord, 2002. 13(3): p. 1938.
17. Vinters, H.V., W.G. Ellis, C. Zarow, B.W. Zaias, W.J. Jagust, W.J. Mack, and H.C. Chui, Neuropathologic substrates of ischemic vascular dementia. J Neuropathol Exp Neurol, 2000. 59(11): p. 931-45.

18. De Reuck, J. and G. Van Maele, Cognitive impairment and seizures in patients with lacunar strokes. Eur Neurol, 2009. 61(3): p. 159-63.

19. Mitasova, A., M. Kostalova, J. Bednarik, R. Michalcakova, T. Kasparek, P. Balabanova, L. Dusek, S. Vohanka, and E.W. Ely, Poststroke delirium incidence and outcomes: validation of the Confusion Assessment Method for the Intensive Care Unit (CAM-ICU). Crit Care Med, 2012. 40(2): p. 484-90.

20. Minett, T.S., J.L. Dean, M. Firbank, P. English, and J.T. O'Brien, Subjective memory complaints, white-matter lesions, depressive symptoms, and cognition in elderly patients. Am J Geri. Psychiatry, 2005. 13(8): p. 665-71. 